\title{
Single-Domain Antibodies for the Detection of SARS-CoV-2 Nucleocapsid Protein
}

George P. Anderson ${ }^{1}$, Jinny L. Liu ${ }^{1}$, Thomas J. Esparza ${ }^{2,3}$, Bruce T. Voelker ${ }^{4}$, E. Randal Hofmann $^{4,5}$, Ellen R. Goldman ${ }^{1 *}$

${ }^{1}$ US Naval Research Laboratory, Center for Biomolecular Science and Engineering, 4555

Overlook Ave SW, Washington, DC 20375, USA

${ }^{2}$ The National Institute of Neurological Disorders and Stroke Intramural Research Program, Laboratory of Functional and Molecular Imaging, Bethesda, MD, USA 20892

${ }^{3}$ Henry M. Jackson Foundation for the Advancement of Military Medicine, Bethesda, MD, USA 20892

${ }^{4}$ U.S. Army Combat Capabilities Development Command, Chemical Biological Center, 8198

Blackhawk Road, Aberdeen Proving Ground, MD, USA 21010

${ }^{5}$ EXCET, Inc., 6225 Brandon Ave \#360, Springfield, VA 22150, USA

*Corresponding author: Email: ellen.goldman@nrl.navy.mil, phone: 202-404-6052

\section{Supporting Information}

Content

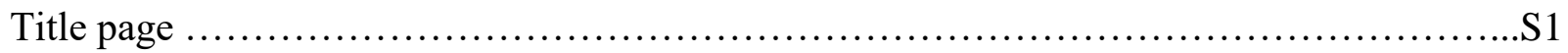

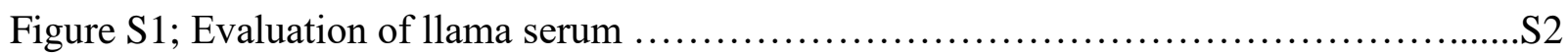

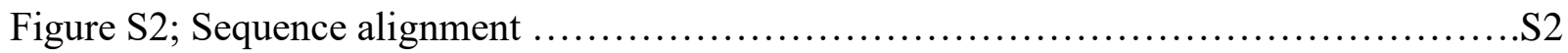

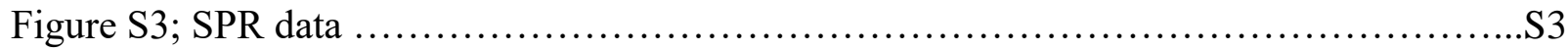

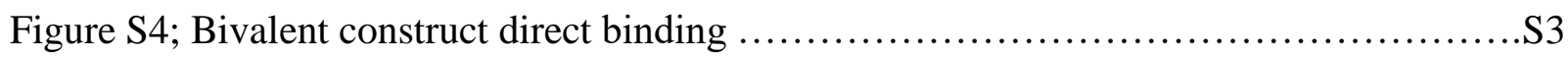

Table S1; SdAb production, melting point and refolding potential......................S4 


\section{Figure S1}

\section{Centavo - Nucleocapsid}

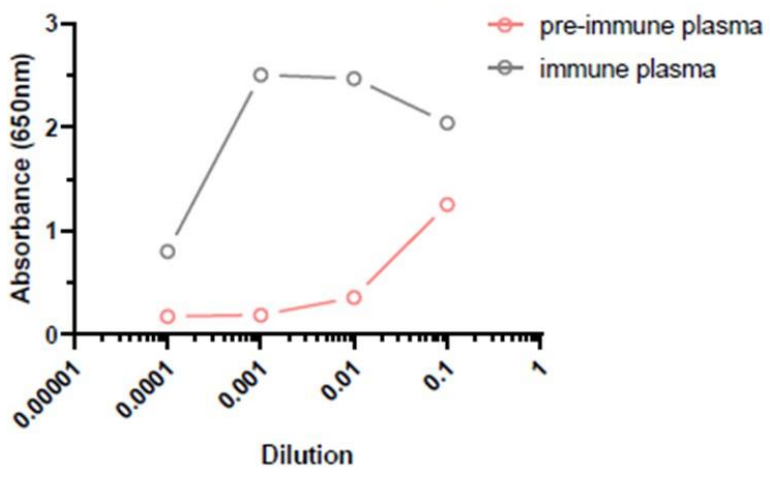

Figure S1. Evaluation of llama serum pre and post immunization for the presence of antibodies towards N by ELISA.

Figure S2

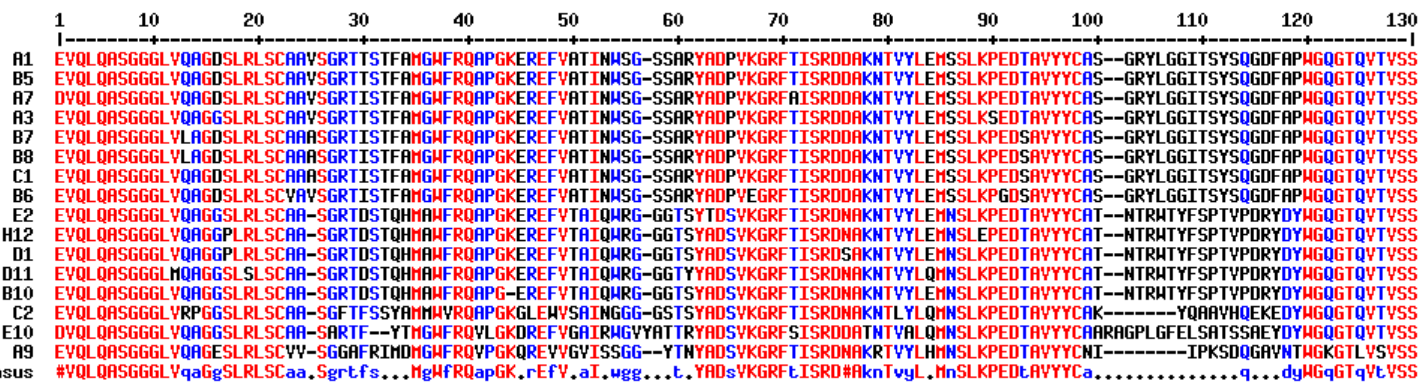

Figure S2. Sequence alignment of the 16 clones identified by the monoclonal phage MagPix assay. Sequences are given in the single letter amino acid code; red denotes high homology position, while lower homology positions are in blue. Positions are numbered sequentially. Using this numbering scheme, we define CDR1 as the region of amino acid residues $26-35$, CDR 2: $50-65$, CDR3: 99 - 119. The clones B6, E2, C2, E10, and A8 were expressed as soluble sdAbs. 


\section{Figure S3}
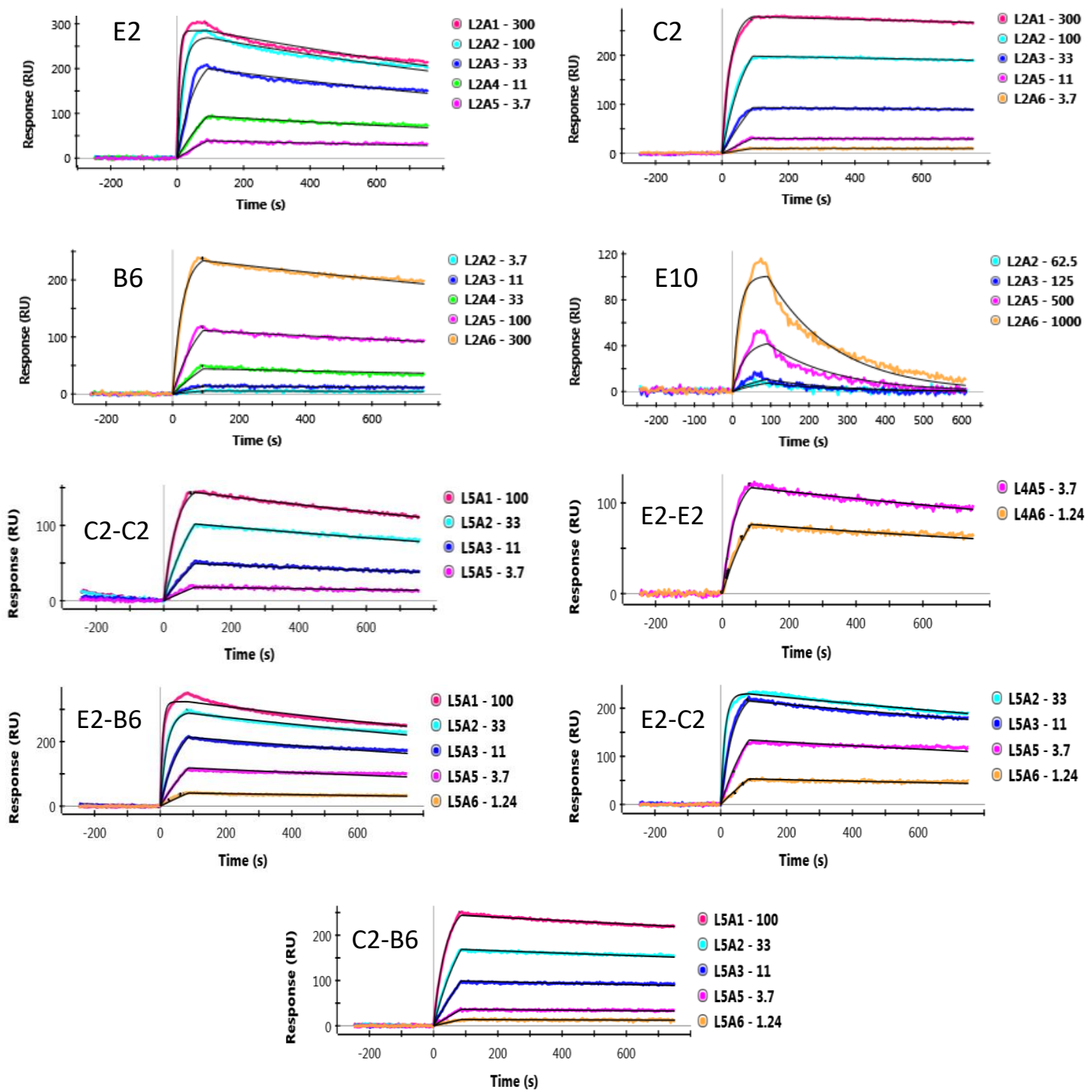

Figure S3. Surface Plasmon Resonance Evaluation of the anti-N sdAbs. Each sdAb was tested by flowing at the concentrations shown (nM) over the surface of a chip with $\mathrm{N}$ immobilized. Only one data set is shown with similar results obtained on all spots and in replicate runs. The data shown has been adjusted by subtraction of both interspot and buffer control responses, the black lines show the data fits used to calculate the kinetic constants shown in Table 1. 


\section{Figure S4}

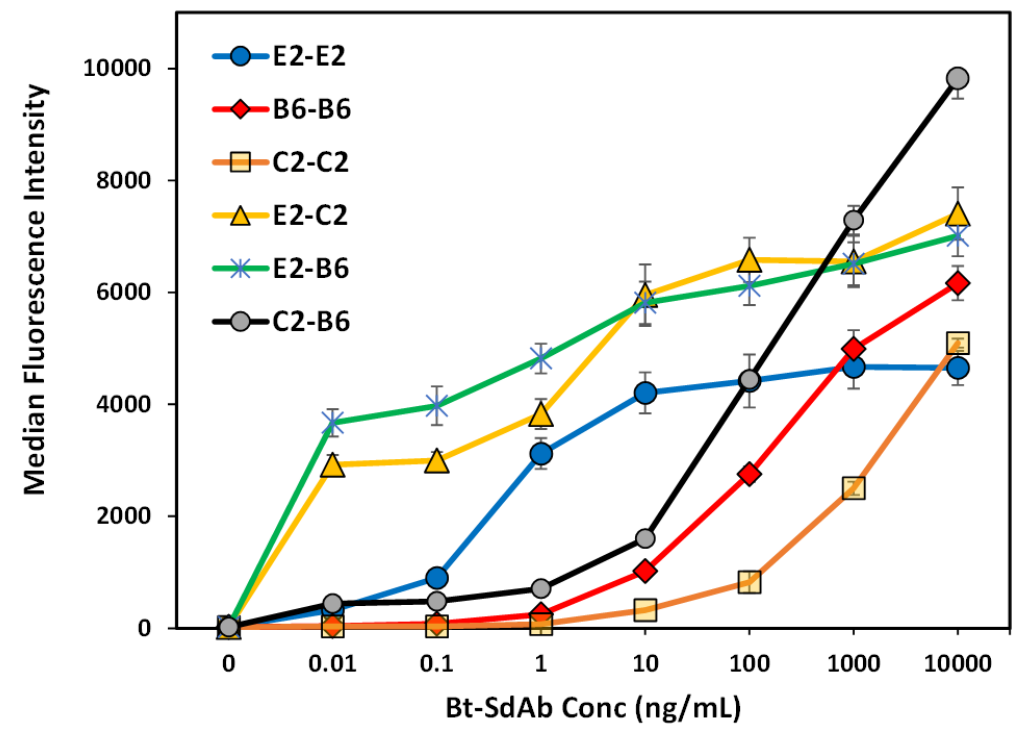

Figure S4. Direct binding data of biotinylated bivalent constructs of the sdAbs to MagPlex microspheres coated with $\mathrm{N}$. Errors bars represent the standard error of the mean of two sets of $\mathrm{N}$ microspheres evaluated. Not shown are RBD coated microsphere to which little to no binding was observed.

\section{Table S1}

Table 1: SdAb production, melting point and refolding percentage

\begin{tabular}{|c|c|c|c|}
\hline sdAb & Yield (mg/L) & $\operatorname{Tm}(\mathrm{CD})$ & \% refold \\
\hline C2 & 14 & 67.5 & 63 \\
\hline C2-hop & 22.5 & 65 & 56 \\
\hline E2 & 30.4 & 62 & 50 \\
\hline E2-hop & 36.7 & 62 & 44 \\
\hline E10 & 7 & 70 & 41 \\
\hline E10-hop & 4.6 & 68 & 47 \\
\hline B6 & 2 & 55 & 87 \\
\hline B6-hop & 2.6 & 55 & 62 \\
\hline
\end{tabular}

